\title{
Design of the control system of the bidirectional DC/DC converter for the storage and regeneration
}

\author{
Deng Yonghong, Ge Yanxiang, Qian Huifa \\ College of electronic and information engineering, North China Institute of Science and Technology, 065201
}

\begin{abstract}
In view of the phenomena of energy waste, environmental pollution and increase of operating cost caused by the braking of the rail transport vehicle, the storage and regeneration of bidirectional DC/DC converter control system is designed, It takes TMS320F28035 DSP as the core control circuit, and adopts voltage and current double closed loop PI control. The system realizes the storage and regeneration of energy during operation. The experiment shows that the system has high reliability and anti-interference ability, so it can be popularized and applied.
\end{abstract}

\section{Introduction}

The accumulator locomotive is powered by the storage battery, so it is suitable for transportation in the tunnel with more gas and coal dust accumulation, safety-type battery locomotives can also operate safely in the main ventilation tunnel of super gas mines. As the traditional traction power supply system can not reverse recovery energy, the energy generated by the regenerative braking of the traction tractor is not only wasted, but also increased the burden of other electrical equipment operation, and increasing the ventilation system load. Similarly, urban rail vehicles also have this problem. This paper studies energy storage and regeneration technology of rail vehicles, and make reasonable use of the energy produced by braking, to achieved the purpose of saving energy, improving carrying capacity and stabilizing the supply voltage of a storage battery or a power supply network. Using super capacitor bank as the scheme of accumulator. At present, there is no mature product in the domestic application, the regenerative braking energy of super capacitor adopt in domestic research has just started, and it has just started in urban rail transportation. In coal mine, it is of great practical significance to study the storage and regenerative bidirectional DC/DC converter control system in track operation.

\section{Constitution and principle of control system}

In orbit, the structure of a power supply system incorporating a bidirectional DC-DC converter is shown in figure 1. The system consists of a storage battery or a power supply network, a bidirectional DC-DC converter, a super capacitor set, an inverter and a He Yongci synchronous machine. In the process of vehicle braking charging, the average charging current is determined according to the regenerative braking power and the capacitor voltage of the locomotive, and the charging process can be automatically ended when the super capacitor voltage reaches the highest charging voltage. In the discharge process, the discharge current is determined according to the starting power of the vehicle and the voltage of the super capacitor, and the bidirectional DC-DC converter is turned off when the voltage of the ultracapacitor is reduced to the minimum operating voltage.Whether the converter operating condition is the charging state or the discharging state, It is determined by the conditions of the vehicle operation, the voltage of the power supply network and the super capacitor voltage. In the case of running conditions, controling the control signal of the main circuit of the corresponding contactor controlchip and a certain duty cycle PWM pulse,which were sent by high-speed 
output port of control chip, and by the drive circuit to isolate the signal after the drive, the main circuit switching device IGBT Work according to the required duty cycle. thus achieve the recovery of braking energy and reuse.

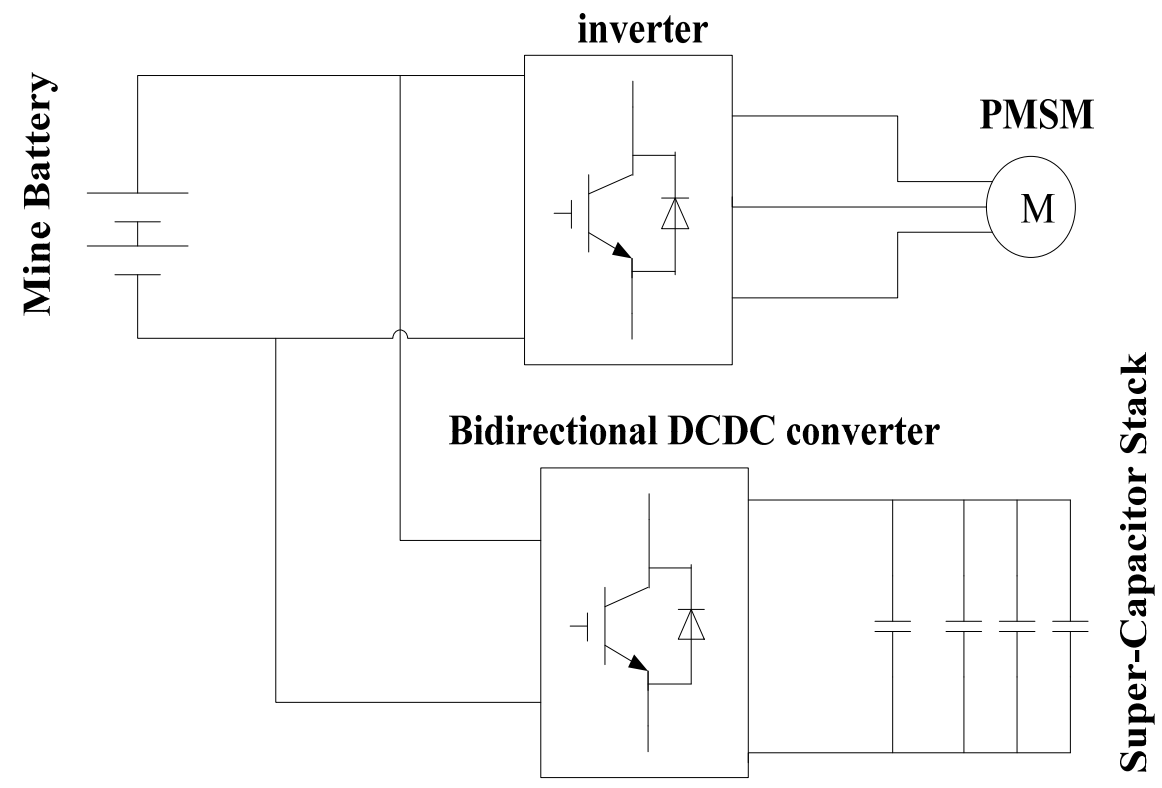

Figure 1 Mine electrical locomotive power supply system structure

\subsection{Analysis of operation principle of bidirectional DC/DC converter}

As shown in Fig. 2, The bidirectional DC/DC converter consists of a three-phase three chopper circuit, the difference of control pulse is 120 degrees, and its working principle is as follows:

(1) when the vehicles brake again, resulting the power grid voltage increased, bidirectional DC/DC converter circuit in Buck circuit model, and the super capacitor energy storage system connected, for charging. The ultracapacitor group absorbs regenerative braking energy while reducing the voltage of the power supply network.

(2) when the vehicle is accelerating or starting, power supply network voltage will be lower, then the controller of bi-directional $\mathrm{DC} / \mathrm{DC}$ converter working in Boost circuit mode, super capacitor energy feedback through bidirectional converter. Under this condition, the super capacitor energy storage system is put into use, which reduces the requirement of the peak power of the power supply system, and makes the voltage drop of the power supply network be eased.

(3) when the running vehicle is idle, the controller can control the energy storage state of the super capacitor set by the main circuit of the bidirectional DC/DC converter according to the capacitance of the super capacitor group.

To sum up the analysis can be seen, according to the different operation condition of the vehicle and power supply voltage, the controller control bidirectional DC/DC converter to achieve super capacitor energy storage system and inverter energy exchange, and achieve energy storage utilization. 


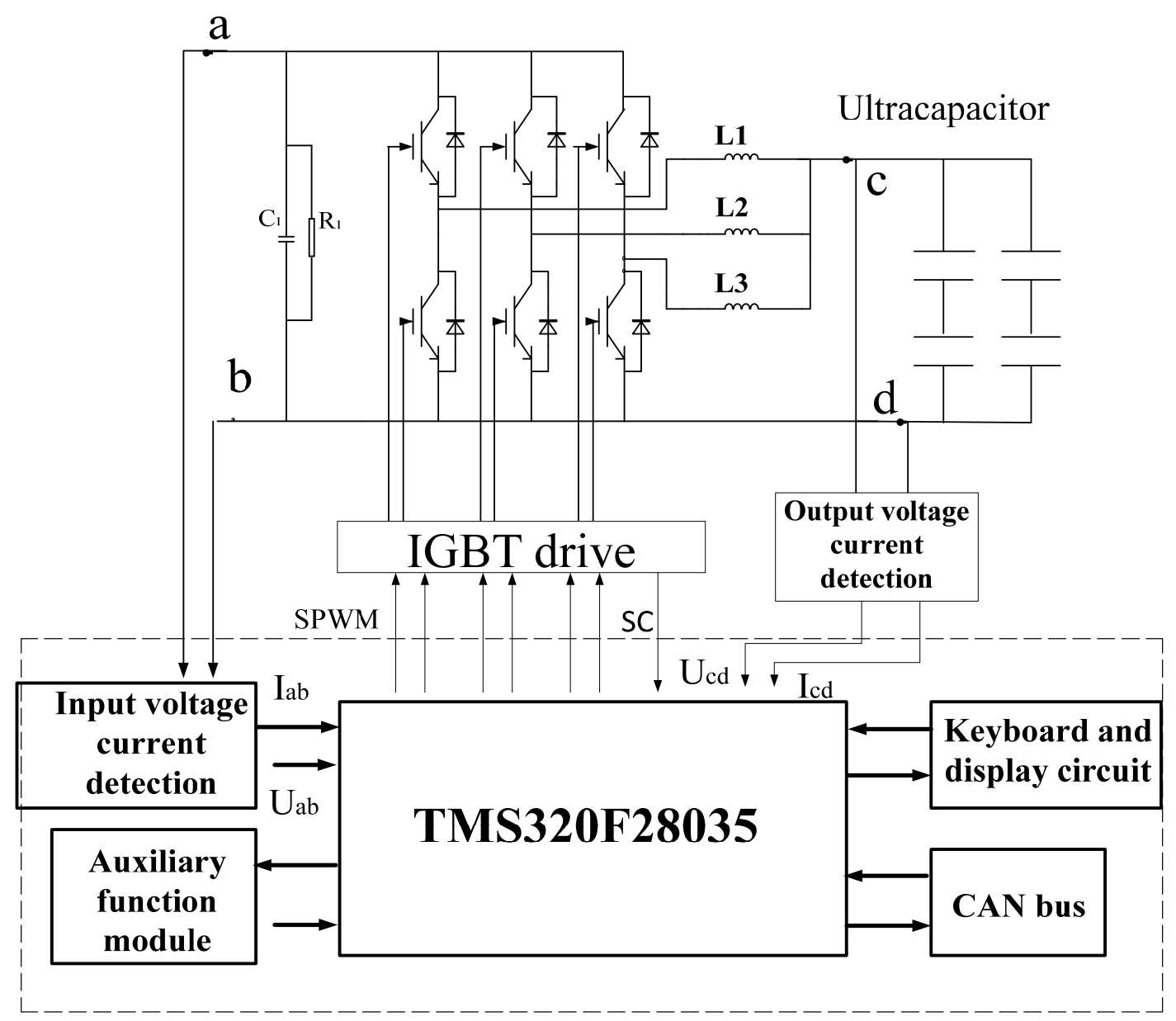

Fig. 2 overall structure diagram of the system

\section{DSP control block diagram}

The TMS320F28035 control circuit is the core part of the system, and its block diagram is shown in figure 3 . The system consists of five parts, such as DSP digital system, touch sensitive interface circuit, IGBT drive circuit, CAN communication circuit and keyboard display circuit. The functions of each part are as follows:

(1) the main task of DSP digital system is receiving the upper control computer or run vehicle bus from the given conditions according to the given information and instructions, Then according to the given, To compare and calculate the physical quantities such as voltage, current with setting value, the output PWM switch control pulse and the signal control system;

(2) analog analog interface circuit converts the strong current signal into weak current signal for digital sampling of DSP;

(3) the IGBT drive circuit converts the DSP system's signal into the pulse signal that can drive IGBT, and meanwhile, when the IGBT has short circuit or over-current fault, it sends back the fault signal to the DSP system;

(4) the function of the keyboard display circuit is to set various instructions and parameters through the keyboard, display the various states of the system operation, and record the system history running fault. 


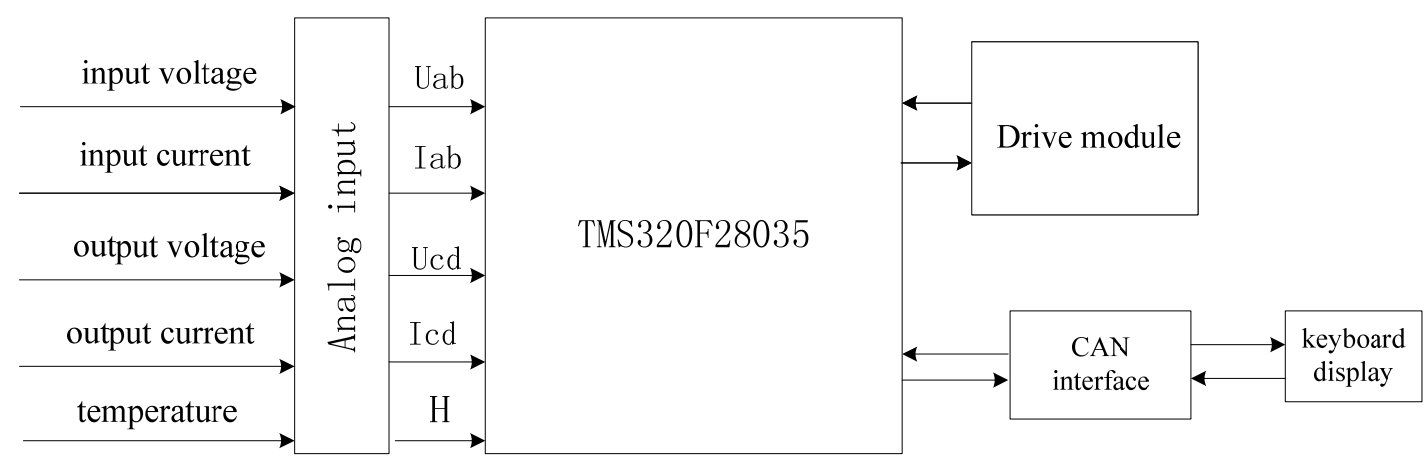

Fig. 3 block diagram of control system

\section{Control Algorithm and Software Design}

\section{1 control algorithm analysis}

Block diagram of control algorithm is used in the system, as shown in Figure 4 (a), its working principle is as follows: the feedback value of output voltage $U_{1}$ of the power supply system is different from the given reference value $U_{\text {ref }}, U_{1}$ through the PI regulator as the given current signal output. The current command $\mathrm{I}_{\text {ref }}$ is different from the inductance current $\mathrm{i}_{\mathrm{L}}$, and finally get the PWM signal of Power tube VT_1 of the Upper bridge arm after PI adjustment; maximum $\mathrm{U}_{2 \max }$ and minimum $U_{2 \min }$ of allowable voltage compared to the voltage $U_{2}$ of super capacitor, the voltage amplitude limiting closed loop of ultra capacitor side is formed, closed limit the highest limit voltage loop and minimum voltage respectively .Comparing the current $i_{\text {ref1 }}$ of regulator output with the Limiting amplitude of Maximum current $I_{\max }$ and minimum current $I_{\min }$, the current command $i_{\text {ref1 }}$ gets the $I_{\text {ref }}$ after the clipping closed loop, and the $\mathrm{I}_{\text {ref }}$ is used as the input value of the current closed loop.

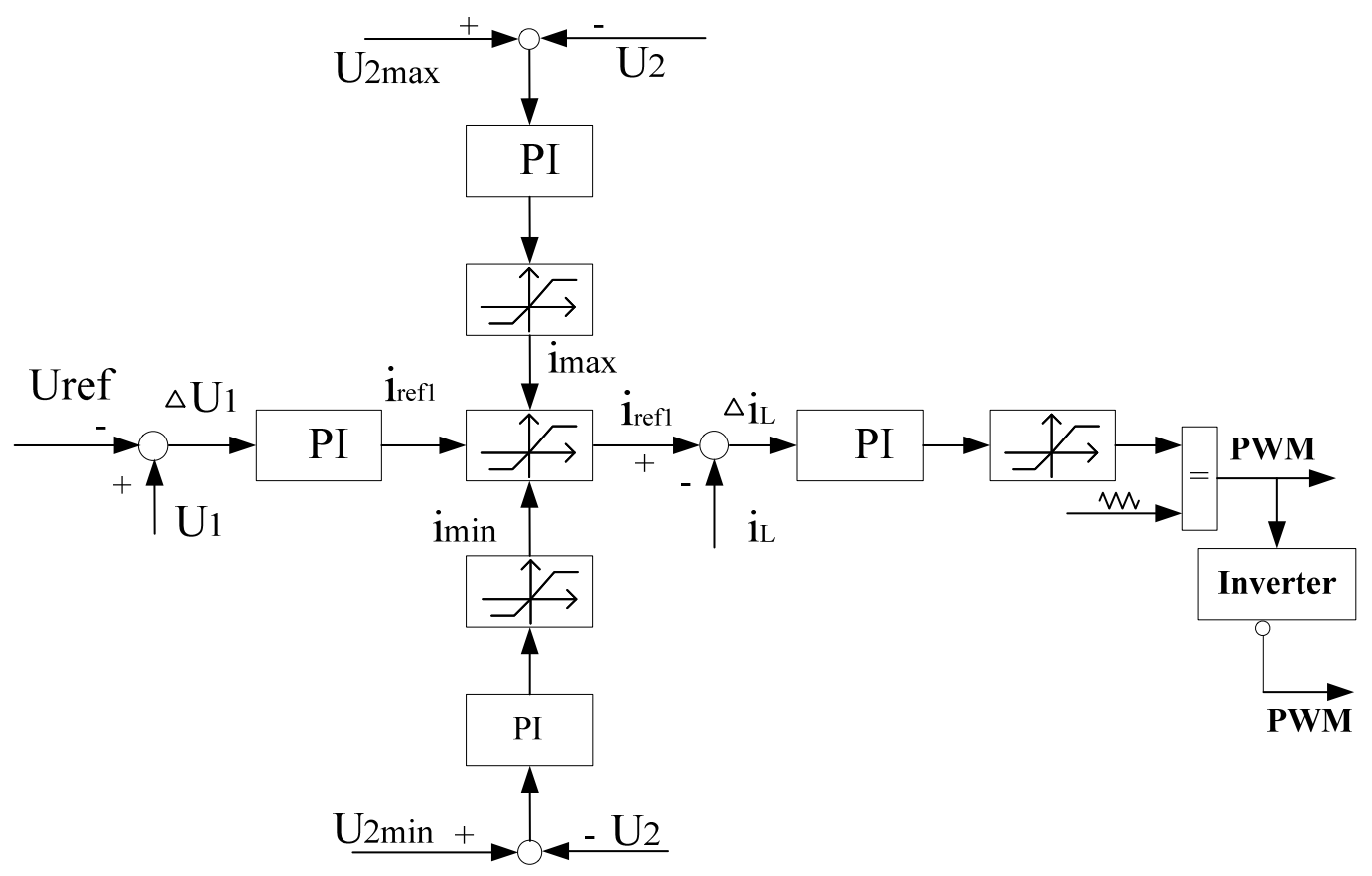

Fig. 4 (a) block diagram of bidirectional DC-DC control algorithm 


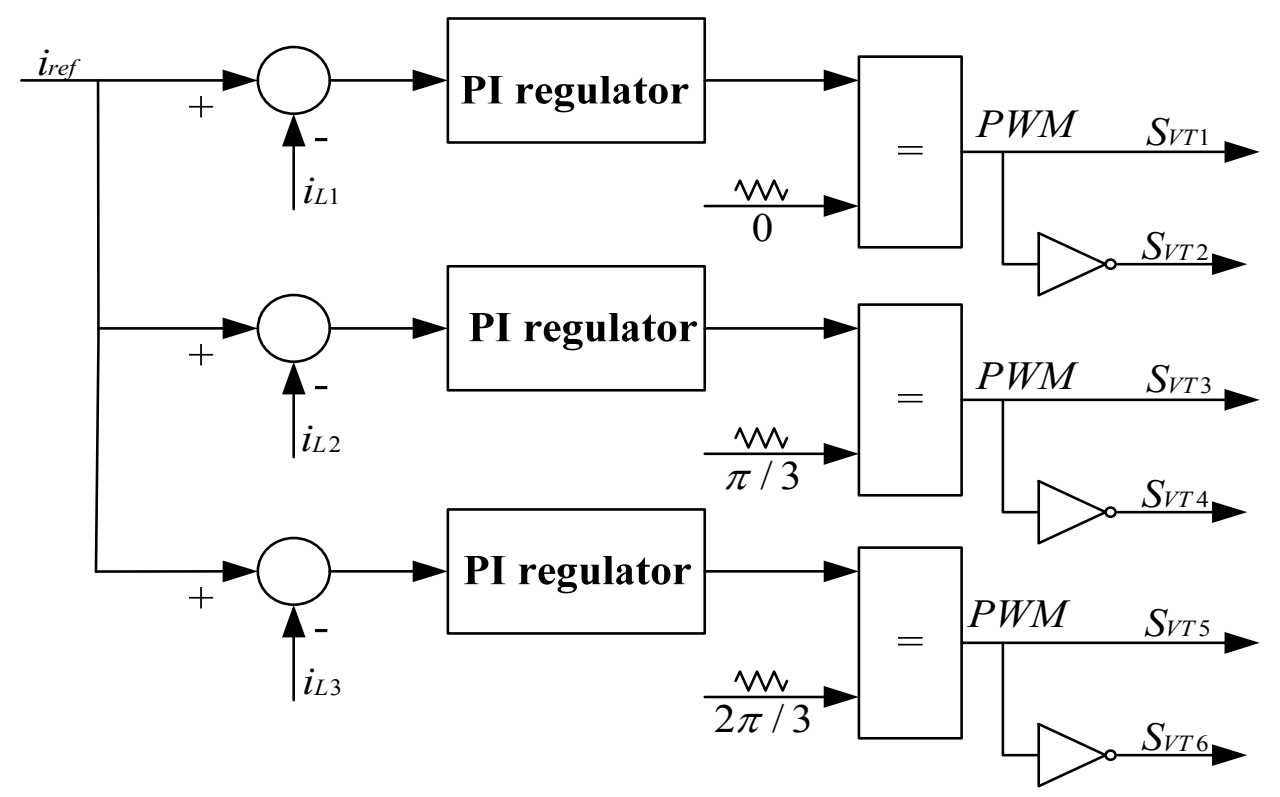

Figure 4 (b) closed-loop current sharing control diagram

Figure 4 shows that the voltage and current double PI closed loop control algorithm, through the DSP to calculate the value of the PI regulator, the output difference of 120 degrees of PWM wave control, three-phase three - way bi-directional DC-DC converter.

\section{2 software design}

As shown in Figure 5, the software program diagram of the system is composed of main program, interrupt service program, CAN interface program and $\mathrm{A} / \mathrm{D}$ interface program.

Then the interrupt get into circulation, waiting for DSP initialization, clock circuit configuration, the peripheral circuit and software initialization variables in the definition and assignment, system interrupt configuration of system initialization task; then open communication interrupt, accept the control signal of the upper computer or the inverter controller carries out data transmission, To enable the system to get boot instructions or super capacitor charge and discharge instructions.

The interrupt service program completes all control algorithms, such as current and voltage detection, A/D converter, closed loop PI control and PWM occur, The program sets up three interrupts: interrupts, reads, sampling results, interrupts, and fault protection interrupts, As mentioned in the preceding paragraph, Compare interrupt is used to generate control cycles periodically, and A/D sampling completes sampling results after reading.

The CAN interface program simply passes the status data and control instructions. The basic steps include initialization of the CAN module, sending data, receiving data, and checking the correctness of the data. The A/D interface program completes the frequency used by $\mathrm{AD}$ and sets the sampling mode. 


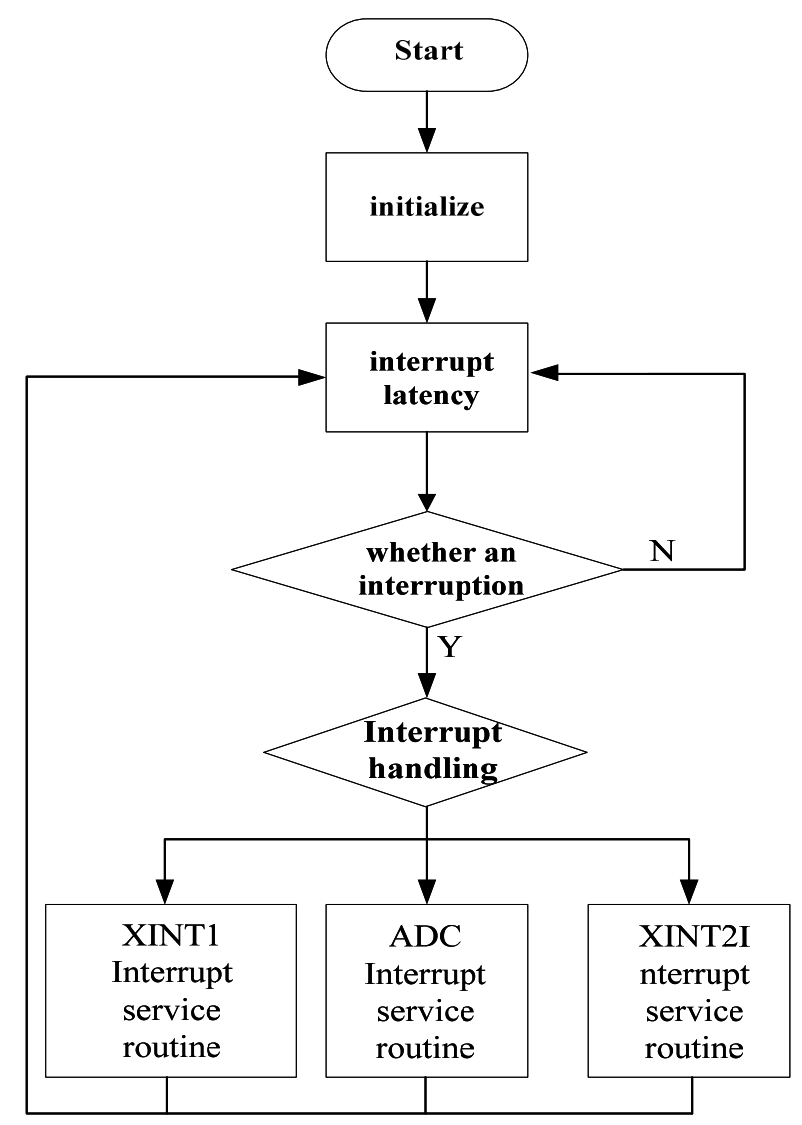

Fig. 5 program flow chart

\section{4 experiment debugging and result analysis}

The bidirectional DC-DC converter system has been successfully implemented. The main parameters of the system are as follows: the voltage range of the power grid is between DC163.2V and DC288V, the actual test was DC210V at the time of the experiment, rated power of permanent magnet synchronous shock motor is $22 \mathrm{~kW}$ * 2. Bidirectional DC-DC converter parameter: The value of the inductor is $2.8 \mathrm{mH}$. The ultracapacitor group is $60 \mathrm{~V} / 125 \mathrm{~F}$, two series connected, set the PWM frequency of the control system to $5 \mathrm{~K}$. Carry on load test, charge the super capacitor when the vehicle slows down or stop. Through the buck chopper circuit, the constant current charging of the bidirectional DC-DC converter.
When the motor vehicle accelerates or starts, the super capacitor is supplied to the inverter through a boost chopper circuit, and the actual voltage is 195V. Figure 6 shows the DSP output PWM debug waveform, and the bi-directional DC-DC converter system is stable.

\section{Conclusion}

Energy storage and regeneration bidirectional DC-DC converter system is Realized for rail vehicles, and the current and voltage double closed loop PI regulator control system has been successfully developed based on DSP. The hardware of the system is simple, modular strong, reliable operation, the recovery and reuse of brake energy are realized. The system has better engineering practicability and popularization value. 

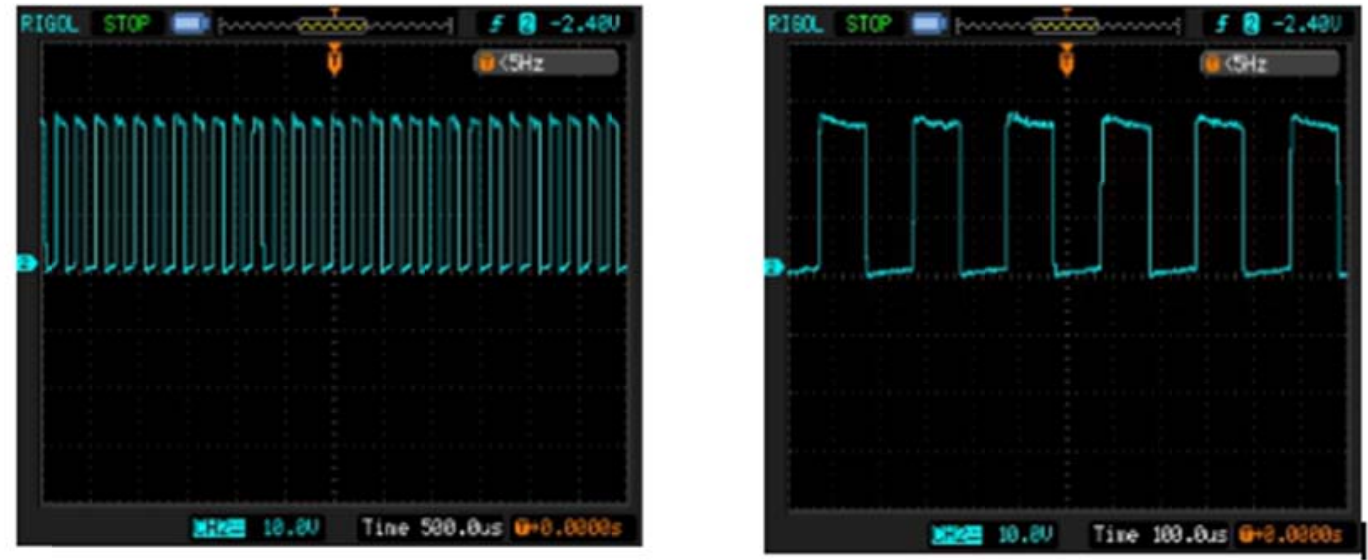

\section{Figure 6 PWM debug waveform}

\section{Reference:}

[1] Wang Zhaoan, Huang Jun. Power electronic technology. Fourth edition. Beijing: China Machine Press, 2006

[2], Pan Xinmin, Wang Yanfang. Practical tutorial of microcomputer control technology. First edition. Beijing: Publishing House of electronics industry, 2010

[3] Liu Lingshun, high bright, Zhang Shutuan, Wang Fang.TMS320F28335DSP principle and development programming. Beijing: Beihang University press, 2011

[4] Chen Boshi. Electric drive automatic control system. Third edition. Beijing: Mechanical Industry Press, 2011

[5] Hu Shousong. The principle of automatic control. Fifth edition. Beijing: Science Press, 2007

[6] $\mathrm{Xu}$ Dehong. Modeling and control of power electronic systems. First edition. Beijing: China Machine Press, 2006

[7], Wang Xinggui, Zou Yingwei, Liu Jinlong. Modeling and control of full bridge DC/DC switching power.
Power electronics, 2007.7, 41 (7): 86 88

[8] Cui steel. Research and development of PWM switching power supply based on DSP control: dissertation. Dalian: Dalian University of Technology, 2006.6.1

[9] Deng Yonghong, Zhang Quanzhu, Ren Xuejun, Zhi Qiang. Research on the power supply mode of the class driven offshore oil logging device of motor.

Power technology. 2011, 35 (2): 202-206.

[10] Wang Xuedi. Research on super capacitor energy storage system based on urban rail transit. [Dissertation], Beijing: Beijing Jiaotong University, 2008.6

Foundation item: the project by science and Technology Department of Hebei province (1627460316214408); Chinese Coal Industry Association (MTKJ2016-35, MTKJ2016-329); fundamental research funds for the central universities such as funding (3142016022, 3142017044) 